\title{
A possible reason for the low reproducibility of malondialdehyde determinations in biological samples
}

\author{
"Malondialdehyde is used as a biomarker for many common health \\ problems, such as cancer, cardiovascular, pulmonary, psychiatric and \\ infectious diseases."
}

First draft submitted: 22 August 2016; Accepted for publication: 7 September 2016; Published online: 26 September 2016

Keywords: derivatization $\bullet$ human plasma $\bullet$ malondialdehyde $\bullet$ oxidative stress

- reproducibility $\bullet$ thiobarbituric acid

Malondialdehyde (MDA) is the most frequently used biomarker of oxidative stress (imbalance between oxidant and antioxidant systems) in clinical investigations. MDA is used as a biomarker for many common health problems, such as cancer [1], cardiovascular [2], pulmonary [3], psychiatric [4] and infectious [5] diseases. Although the results of case-control comparisons using statistical analyses have shown significantly increased MDA levels in the case groups, some nonsignificant changes and even some significantly decreased values for MDA levels in case groups against control groups have been observed [6-10]. In addition, there are very wide variations in the reported MDA values in both case and healthy control groups. As an example, reported plasma MDA levels vary from 0.32 [11] to $53.8 \mathrm{nmol} / \mathrm{ml}$ [4]. A quick survey of the MDA levels of biological samples measured using a single analytical method revealed that there is huge variability in the MDA levels of healthy people [12]. The variations are wider when various analytical methods are considered [6]. Proposed reasons for these variations include the following:

- Variations in the production rate of MDA in biological fluids

- Effects of diet and physical activity

- Effects of sampling time

- Effects of sample storage conditions
- High reactivity of MDA and crossreactions with biochemicals present in the biological samples

- Variations in derivatization rate

- Variations owing to the analytical methods

These points along with some further aspects were recently reviewed and readers are referred to [13] for further details.

Formation of an adduct with thiobarbituric acid (TBA) and its spectrophotometric determination is the most commonly used analytical procedure for determination of MDA in biological samples. Despite the poor selectivity of spectroscopic determination of MDA, it is still widely used even in recent research works. Another main problem with the method is its low reproducibility, which is caused by different factors as follows [14-17]. Derivatization steps of most of the reported methods were carried out at temperatures of higher than $70^{\circ} \mathrm{C}$ and usually $100^{\circ} \mathrm{C}$. The boiling point of $\mathrm{MDA}$ is $109^{\circ} \mathrm{C}$ so at higher temperatures, evaporation of MDA is possible and its concentration could be changed owing to the evaporation of MDA or water. Concerning this hypothesis, the effects of possible evaporation on the reproducibility of MDA determination have been investigated. Based on derivatization conditions in plasma, including reflux, open and closed systems, reproducibility of the results was
Sajad Azizi

Pharmaceutical Analysis Research Center \& Faculty of Pharmacy, Tabriz University of Medical Sciences, Tabriz 51664, Iran

\section{Aziz Shahrisa}

Department of Organic \& Biochemistry, Faculty of Chemistry, University of Tabriz, Tabriz 51666, Iran

Maryam Khoubnasabjafari Tuberculosis \& Lung Disease Research Center, Tabriz University of Medical Sciences, Tabriz 51664, Iran

Khalil Ansarin

Tuberculosis \& Lung Disease Research Center, Tabriz University of Medical Sciences, Tabriz 51664, Iran

Mehry Khoubnasabjafari Liver \& Gastrointestinal Diseases Research Center, Tabriz University of Medical Sciences, Tabriz 51664, Iran

\section{Jafar Soleymani}

Drug Applied Research Center, Tabriz University of Medical Sciences, Tabriz 51664, Iran

\section{Abolghasem Jouyban}

Author for correspondence: Pharmaceutical Analysis Research Center \& Faculty of Pharmacy, Tabriz University of Medical Sciences, Tabriz 51664, Iran

and

Kimia Idea Pardaz Azarbayjan (KIPA) Science Based Company, Tabriz University of Medical Sciences, Tabriz 51664, Iran

Fax: +98 4133363231

ajouyban@hotmail.com

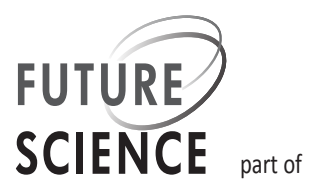


For reprint grders, please contact reprints@future-science.com

all samples, a pooled plasma sample was used [Azızı S, Khoubnasabjafari M, Jouyban A, Unpublished Data].

Temperature is considered as a major factor that affects the rate of a chemical reaction. Keeping the $\mathrm{pH}$ value of the reaction solution at 2.5 and the ratio of $\mathrm{TBA} / \mathrm{MDA}$ at 5 , the effect of temperature was investigated at three temperatures: 70, 85 and $100^{\circ} \mathrm{C}$. Previous studies showed that MDA analysis is affected by the solution temperature at the derivatization stage, the $\mathrm{pH}$ of the solution and the molar ratio of TBA/MDA [13,18-19]. According to our observations [Azizi S, Khoubnasabjafari M, Jouyban A, Unpublished Data], the absorbance is increased remarkably by setting the reaction temperature to $70^{\circ} \mathrm{C}$. In addition, the temperature of the reaction affects both repeatability and reproducibility. Concerning the boiling point of MDA (approximately $109^{\circ} \mathrm{C}$ ), at higher temperatures evaporation of MDA may also cause the loss of analyte and subsequently a decrease in the absorbance of the solution. Production of MDA from lipid peroxidation and its release from proteins are affected by temperature and may cause poor reproducibility. It is obvious that at lower temperatures the reaction rate will be decreased, and a longer reaction time is required for derivatization of MDA. We found that $180 \mathrm{~min}$ is a reasonable time for the derivatization stage.

\section{"Analysis of malondialdehyde as a biomarker of oxidative stress is an interesting subject in medi- cal investigations since oxidative stress is one of the well-accepted causes..."}

In a series of experiments conducted under the same conditions in open, closed and refluxed systems, the observed UV absorbance of MDA-TBA was higher in open and closed systems than in the refluxed system. The sampling times were 30, 60, 90, 120, 150 and $180 \mathrm{~min}$. Higher absorbance and a greater reduction in solution volume were observed for the open system along with the highest variations in the absorbance, revealing evaporation of MDA and water. In the closed system, both evaporations are decreased but the absorbance variations are high [Azizi S, Khoubnasabjafari M, Jouyban A, Unpublished Data]. The refluxed system showed better repeatability. It seems that poor reproducibility and repeatability as the main drawbacks of the spectroscopic methods [20] are resolved in the refluxed system where the relative standard deviations for all data points are between 0.7 and $6.1 \%$ (average, 2.8\%), while the relative standard deviations in the open and closed systems are 23 and $21 \%$, respectively. Therefore, the reflux procedure is recommended for determination
Of MDA in biological samples to provide acceptable reproducibility and repeatability of the analytical results.

Analysis of MDA as a biomarker of oxidative stress is an interesting subject in medical investigations since oxidative stress is one of the well-accepted causes, or at least an initial cause, of the most frequent health problems. Unfortunately, most MDA measurements are carried out without prior validation of the methods used in the studies. We were not able to obtain acceptable validation data using several previously established analytical methods employing spectrophotometric and spectrofluorometric detection methods, even after liquid chromatographic separations. Careful review of the literature revealed that a number of research groups pointed out such poor validation data [14-17]; however, these important and critical points have been ignored by research groups, and simple derivatization and spectrophotometric determination of MDA is frequently used in medical investigations, as correctly stated by Wade $\&$ van Rij [21]. The rapidity, ease of use and very low cost made spectroscopic assay of MDA-TBA adduct a popular analytical method for monitoring MDA levels in biological samples, and its limitations were simply ignored by most of biomedical researchers. Our results revealed that to reach a more reproducible and repeatable procedure, the derivatization reaction should be performed in a refluxed system at $70^{\circ} \mathrm{C}$ at $\mathrm{pH}$ 2.5. Using these reaction conditions, MDA could be analyzed reproducibly in human plasma samples. It is obvious that to improve the selectivity of the spectroscopic method, a separation method should be combined with the analytical method and full validation data should be obtained prior to application of the method for analysis of biological samples. A valid analytical method should be employed for quantification of MDA in biological samples and it is strongly recommended to not use nonvalidated analytical methods in clinical investigations to avoid misleading by the obtained results and, consequently, controversial findings in health/disease conditions.

\section{Financial \& competing interests disclosure}

The authors have no relevant affiliations or financial involvement with any organization or entity with a financial interest in or financial conflict with the subject matter or materials discussed in the manuscript. This includes employment, consultancies, honoraria, stock ownership or options, expert testimony, grants or patents received or pending, or royalties.

R De Vooght-Johnson (Freelance Scientific Writer and Editor [Royston, UK]) proofread draft of the manuscript and the language edit payment has been made by Tabriz University of Medical Sciences. 


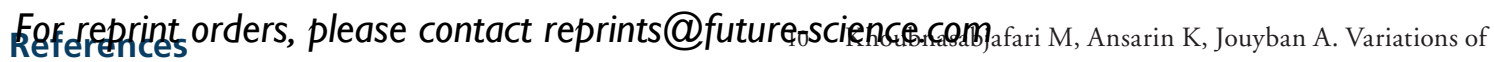

Papers of special note have been highlighted as:

- of interest; $\bullet \bullet$ of considerable interest

$1 \quad$ Kilic N, Taslipinar MY, Guney Y et al. An investigation into the serum thioredoxin, superoxide dismutase, malondialdehyde, and advanced oxidation protein products in patients with breast cancer. Ann. Surg. Oncol. 21(13), 4139-4143 (2014).

2 Vanishree BJ, Kodliwadmath MV, Prasad CVB, Sonoli SS. Malondialdehyde, ascorbic acid and lipid profile in diabetic and hypertensive with myocardial infarction: a case control study. Biomedicine 31(2), 231-234 (2011).

3 Antus B, Drozdovszky O, Barta I, Kelemen K. Comparison of airway and systemic malondialdehyde levels for assessment of oxidative stress in cystic fibrosis. Lung 193(4), 597-604 (2015).

4 de Sousa RT, Zarate CA, Zanetti MV et al. Oxidative stress in early stage bipolar disorder and the association with response to lithium. J. Psych. Res. 50, 36-41 (2014).

5 Sönnerborg A, Carlin G, Akerlund B, Jarstrand C. Increased production of malondialdehyde in patients with HIV infection. Scand. J. Infect. Dis. 20(3), 287-290 (1988).

6 Khoubnasabjafari M, Ansarin K, Jouyban A. Comments on 'An investigation into the serum thioredoxin, superoxide dismutase, malondialdehyde, and advanced oxidation protein products in patients with breast cancer'. www.surgonc.org/news-publications

- A short survey on malondialdehyde (MDA) variations in control group reported by 20 different research groups.

7 Khoubnasabjafari M, Ansarin K, Jouyban A. Comments concerning 'Comparison of airway and systemic malondialdehyde levels for assessment of oxidative stress in cystic fibrosis'. Lung 193(5), 867-868 (2015).

- A short survey on MDA variations in pediatrics.

8 Khoubnasabjafari M, Ansarin K, Jouyban A. Reliability of malondialdehyde measurements as a marker of oxidative stress in pediatrics. Pediat. Neonatol. doi:10.1016/j. pedneo.2015.10.015 (2016) (Epub ahead of print).

- A short survey on MDA variations in oral diseases.

9 Khoubnasabjafari M, Ansarin K, Vaez-Gharamaleki J, Jouyban A. Comments on 'Salivary 8-hydroxy-2deoxyguanosine, malondialdehyde, vitamin $\mathrm{C}$, and vitamin $\mathrm{E}$ in oral pre-cancer and cancer: diagnostic value and free radical mechanism of action'. Clin. Oral Invest. 20, 395-396 (2016).

- A short survey on MDA variations in pregnancy. malondialdehyde in pre-eclampsia. Hypertens. Pregnancy 35(3), 346-349 (2016).

11 Lee DM. Malondialdehyde formation in stored plasma. Biochem. Biophys. Res. Comm. 95(4), 1663-1672 (1980).

-• A short survey on MDA variations and its reliability in psychological diseases and more details on MDA variations reported by different research groups employing a given analytical method.

12 Khoubnasabjafari M, Ansarin K, Jouyban A. Reliability of malondialdehyde as a biomarker of oxidative stress in psychological disorders. BioImpacts 5(3), 123-127 (2015).

-• A comprehensive review article dealing with the effects of analytical conditions on MDA levels and a brief review on recent analytical methods for determination of MDA in biological samples.

13 Khoubnasabjafari M, Ansarin K, Jouyban A. Critical review of malondialdehyde analysis in biological samples. Curr. Pharm. Anal. 12(1), 4-17 (2016).

14 Kei S. Serum lipid peroxide in cerebrovascular disorders determined by a new colorimetric method. Clin. Chim. Acta 90(1), 37-43 (1978).

15 Kikugawa K, Kojima T, Yamaki S, Kosugi H. Interpretation of the thiobarbituric acid reactivity of rat liver and brain homogenates in the presence of ferric ion and ethylenediaminetetraacetic acid. Anal. Biochem. 202(2), 249-255 (1992).

16 Schoenmakers AW, Tarladgis BG. Reliability of the thiobarbituric acid test in the presence of inorganic iron. Nature 210, 1153 (1966).

17 Stalikas CD, Konidari CN. Analysis of malondialdehyde in biological matrices by capillary gas chromatography with electron-capture detection and mass spectrometry. Anal. Biochem. 290(1), 108-115 (2001).

18 Guillen-Sans R, Guzman-Chozas M. The thiobarbituric acid (TBA) reaction in foods: a review. Crit. Rev. Food Sci. Nutr. 38, 315-330 (1998).

19 Kaykhaii M, Yahyavi H, Hashemi M et al. A simple graphene-based pipette tip solid-phase extraction of malondialdehyde from human plasma and its determination by spectrofluorometry. Anal. Biochem. Chem. 408, 4907-4915 (2016).

20 Pacer Z, Veselkova A, Rath R. Oxidative stress. Experienta 21, 19-23 (1965).

21 Wade CR, van Rij AM. Plasma malondialdehyde, lipid peroxides, and the thiobarbituric acid reaction. Clin. Chem. 35(2), 336 (1989). 